\title{
BMJ Open Longitudinal trends in use and costs of targeted therapies for common cancers in Taiwan: a retrospective observational study
}

\author{
Jason C Hsu, ${ }^{1}$ Christine $\mathrm{Y} \mathrm{Lu}{ }^{2}$
}

To cite: Hsu JC, Lu CY. Longitudinal trends in use and costs of targeted therapies for common cancers in Taiwan: a retrospective observational study. BMJ Open 2016;6: e011322. doi:10.1136/ bmjopen-2016-011322

- Prepublication history and additional material is available. To view please visit the journal (http://dx.doi.org/ 10.1136/bmjopen-2016011322).

Received 29 January 2016 Revised 28 April 2016 Accepted 18 May 2016

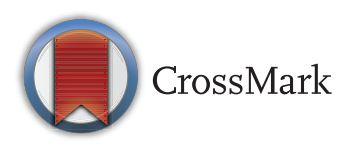

${ }^{1}$ School of Pharmacy and Institute of Clinical Pharmacy and Pharmaceutical Sciences, College of Medicine, National Cheng Kung University, Tainan City, Taiwan

${ }^{2}$ Department of Population Medicine, Harvard Medical School and Harvard Pilgrim Health Care Institute, Boston, Massachusetts, USA

Correspondence to Dr Jason C Hsu; jasonhsuharvard@gmail.com

\section{ABSTRACT}

Objectives: Some targeted therapies have improved survival and overall quality of cancer care generally, but these increasingly expensive medicines have led to increases in pharmaceutical expenditure. This study examined trends in use and expenditures of antineoplastic agents in Taiwan, and estimated market shares by prescription volume and costs of targeted therapies over time. We also determined which cancer types accounted for the highest use of targeted therapies.

Design: This is a retrospective observational study focusing on the utilisation of targeted therapies for treatment of cancer.

Setting: The monthly claims data for antineoplastic agents were retrieved from Taiwan's National Health Insurance Research Database (2009-2012).

Main outcome measures: We calculated market shares by prescription volume and costs for each class of antineoplastic agent by cancer type. Using a time series design with Autoregressive Integrated Moving Average (ARIMA) models, we estimated trends in use and costs of targeted therapies.

Results: Among all antineoplastic agents, use of targeted therapies grew from $6.24 \%$ in 2009 to $12.29 \%$ in 2012 , but their costs rose from $26.16 \%$ to $41.57 \%$ in that time. Monoclonal antibodies and protein kinase inhibitors contributed the most (respectively, $23.84 \%$ and $16.12 \%$ of costs for antineoplastic agents in 2012). During 2009-2012, lung (44.64\% of use; $28.26 \%$ of costs), female breast ( $16.49 \%$ of use; $27.18 \%$ of costs) and colorectal ( $12.11 \%$ of use; $13.16 \%$ of costs) cancers accounted for the highest use of targeted therapies.

Conclusions: In Taiwan, targeted therapies are increasingly used for different cancers, representing a substantial economic burden. It is important to establish mechanisms to monitor their use and outcomes.

\section{INTRODUCTION}

Cancer is a major public health issue globally. Approximately 7.4 million people die of cancer each year worldwide, which accounts for $13 \%$ of all-cause mortality, and

\section{Strengths and limitations of this study}

- This is the first study to examine the national trend in use and costs of targeted therapies for treatment of cancer in Taiwan.

- We also determined which cancer types accounted for the highest use of targeted therapies in Taiwan, from 2009 to 2012.

- Data were retrieved from Taiwan's National Health Insurance Research Database with nearly $99 \%$ of the Taiwanese population (around 23 million residents) enrolled and $97 \%$ of hospitals and clinics throughout the country included.

- A time series design with Autoregressive Integrated Moving Average (ARIMA) models was used in this study, to estimate the trends in market shares by prescription volume and costs of targeted therapies.

- Owing to the lack of patient-level data, this study did not investigate the use of combination treatments; these need to be examined in future studies.

this percentage is expected to increase. ${ }^{1}{ }^{2}$ In Taiwan, cancer is a leading cause of mortality and the annual number of patients with cancer has been growing. ${ }^{3}$ In 2011, 92 682 individuals were diagnosed with cancer (male: 56\%, female: $44 \%$ ). Most common cancers in Taiwan were female breast cancer, colorectal cancer, liver cancer, lung cancer and prostate cancer. In the same year, 42 559 patients died of cancer (male: 64\%, female: $36 \%$ ), accounting for $28 \%$ of all deaths. Major cancers causing mortality were lung cancer, liver cancer, colorectal cancer, female breast cancer and oral/pharyngeal cancer. $^{3}$

Cancer care has improved substantially and the average life expectancy has increased in the past two decades, due to preventative strategies, ${ }^{4}$ early diagnosis, ${ }^{5}$ advances in medical technologies (including surgery and medications $)^{6}$ and clinical management. 
Traditionally, chemotherapies are the main medicines for cancer. But these drugs are not specific to the target, and therefore often cause serious adverse effects including neutropaenia, anaemia and thrombocytopaenia. ${ }^{7}$ In the last decade, however, many new anticancer drugs, so called targeted therapies, ${ }^{8}$ have become available. These drugs differ from standard chemotherapy in that they target specific vulnerable nodes in molecular pathways; ${ }^{9} 10$ thus, they are generally less toxic than traditional chemotherapies. ${ }^{11}$ For some cancers, targeted therapies are becoming the main treatments, for example, trastuzumab for early-stage and human epidermal growth factor receptor 2 (HER2) positive metastatic breast cancer. ${ }^{12} 13$ Dozens of targeted therapies have become available in recent years and many are in the drug development pipeline. ${ }^{14}$ While some have demonstrated improvements in progression-free survival, other agents have provided minimal or no gains in overall survival; for instance, sorafenib, sunitinib, temsirolimus, everolimus, bevacizumab, pazopanib and axitinib for renal cell cancer. ${ }^{15}$

Changes in the cancer treatment paradigm are accompanied by significant economic consequences. Targeted therapies are expensive, typically costing from US $\$ 4500$ to $>$ US $\$ 10000$ per treatment month, even if they demonstrate only improvements in progression-free survival without marked gains in overall survival. ${ }^{15-20}$ The increasing costs of new targeted cancer therapies have risen 10 times during the last decade. ${ }^{21}$ Given the number of new cancer medicines in development and likely continual increases in drug prices, pricing of new anticancer drugs is a real concern for accessibility and affordability across all countries. ${ }^{15} 2223$ Some have suggested that a minimum of improvement in median survival of at least 3-6 months by new cancer medicines compared with current standards is required for the new agent to be considered as advanced and funded at higher prices. ${ }^{24}$ Furthermore, because of the much higher costs of targeted therapies compared with conventional chemotherapy - while the number of eligible patients (due to molecular subtyping) for individual agents is generally small-in aggregate, costs of targeted therapies as a group is an important contributor to growing expenditures for cancer treatments and an important issue of sustainability for all healthcare systems. ${ }^{25-27}$

Owing to limited financial resources, patient access to targeted therapies has been a struggle in many countries. $^{28}$ Many countries have different ways to kerb the growth of pharmaceutical expenditures in general. Examples include formal health technology assessment (for instance, economic evaluation of new drugs is required by many payers/policymakers such as the National Institute for Health and Care Excellence in the UK $^{29} \quad 30$ and Pharmaceutical Benefits Advisory Committee in Australia ${ }^{31} 32$ to select drugs for coverage), pricing tools such as reference pricing ${ }^{33}$ and high patient cost-sharing (co-payments, co-insurance). ${ }^{34}$ To deal with high drug costs and imperfect evidence at the time of marketing approval, many countries are increasingly adopting patient access schemes (also known as managed entry agreements or risk-sharing arrangements) to enable patient access to needed medicines, while ensuring that financing systems are sustainable. $^{35} 36$ The performance of managed entry agreements, however, is largely unknown because most have not been evaluated. ${ }^{33}$ Major challenges at present for many health systems include determining what proportion of the healthcare budget should be allocated for treatment of cancer, including budget for targeted therapies, and designing and implementing new models for pricing, reimbursement, funding and utilisation decisions for cancer medicines. ${ }^{37}$

In Taiwan, economic evaluation has, since 2007, been part of the health technology assessment to evaluate new drugs, to determine decisions for coverage by the National Health Insurance (NHI) ${ }^{38}{ }^{39}$ In addition, prior authorisation is required for many cancer medicines, especially for targeted therapies with high reimbursement prices. An application for prior authorisation can be made to the NHI system, and the drug will be reimbursed if authorisation is given. ${ }^{40}$ For instance, according to 'Directions of Drug Restricted Benefit for National Health Insurance', two targeted therapies, gefitinib and erlotinib, for treatment of lung cancer, have been reimbursed since 2004 and 2007, respectively. In the beginning, both were restricted to be used as thirdline treatment, that is, patients must first have been treated with platinum and docetaxel or paclitaxel chemotherapy, and must have had locally advanced or metastatic adenocarcinoma of the lung. ${ }^{41}$

Little is known about the utilisation and economic impacts of targeted cancer therapies in Taiwan. The aim of our longitudinal analyses was to address this gap by examining the recent trend in utilisation and expenditures of cancer treatments, including targeted therapies, in Taiwan. We also identified which types of cancer accounted for the highest use of targeted therapies.

\section{METHODS}

\section{Data sources}

Taiwan's National Health Insurance Research Database provided data for this study. The database contains information from a nationwide, mandatory-enrolment and single-payer healthcare system created in 1995. Nearly 99\% of the Taiwanese population (around 23 million residents) is enrolled and this system contracts with $97 \%$ of hospitals and clinics throughout the country. ${ }^{42}$ The NHI covers a wide range of prescription medicines, as well as inpatient and outpatient medical services. ${ }^{43}$ All monthly claims data-including details of prescription and insurer spending-for antineoplastic agents, between 2009 and 2012, were retrieved from Taiwan's National Health Insurance Research Database. The cancer-related prescriptions were identified by International Classification of Diseases, Ninth edition (ICD-9) diagnosis codes for cancer (codes 140-239). 
Table 1 Prescription volume of antineoplastic agents in Taiwan (2009-2012)

Number of prescriptions (market share by prescription volume)

2009-2012

\begin{tabular}{|c|c|c|c|c|c|c|c|c|c|c|c|}
\hline \multirow[b]{2}{*}{ Drug class } & \multirow[b]{2}{*}{ Drug name for patients with cancer } & \multicolumn{2}{|l|}{2009} & \multicolumn{2}{|l|}{2010} & \multicolumn{2}{|l|}{2011} & \multicolumn{2}{|l|}{2012} & \multicolumn{2}{|l|}{ 2009-2012 } \\
\hline & & $\mathbf{N}$ & $\begin{array}{l}\text { Per } \\
\text { cent }\end{array}$ & $\mathbf{N}$ & $\begin{array}{l}\text { Per } \\
\text { cent }\end{array}$ & $\mathbf{N}$ & $\begin{array}{l}\text { Per } \\
\text { cent }\end{array}$ & $\mathbf{N}$ & $\begin{array}{l}\text { Per } \\
\text { cent }\end{array}$ & $\begin{array}{l}\text { Growth rate } \\
\text { of } N(\%)\end{array}$ & $\begin{array}{l}\text { Growth rate of } \\
\text { market share } \\
(\%)\end{array}$ \\
\hline All antineoplastic agents & & 1893439 & 100 & 2033160 & 100 & 2300629 & 100 & 2489973 & 100 & 31.51 & \\
\hline Targeted therapies & & 118186 & 6.24 & 150401 & 7.40 & 209030 & 9.09 & 306140 & 12.29 & 159.03 & 6.05 \\
\hline Monoclonal antibodies & $\begin{array}{l}\text { Rituximab, trastuzumab, cetuximab, } \\
\text { bevacizumab }\end{array}$ & 52073 & 2.75 & 68595 & 3.37 & 102074 & 4.44 & 144234 & 5.79 & 176.98 & 3.04 \\
\hline Protein kinase inhibitors & $\begin{array}{l}\text { Imatinib, gefitinib, erlotinib, sunitinib, sorafenib, } \\
\text { dasatinib, nilotinib, temsirolimus, everolimus, } \\
\text { pazopanib }\end{array}$ & 63936 & 3.38 & 78675 & 3.87 & 102435 & 4.45 & 153764 & 6.18 & 140.50 & 2.80 \\
\hline $\begin{array}{l}\text { Other targeted therapy } \\
\text { agents }\end{array}$ & Bortezomib & 2177 & 0.11 & 3131 & 0.15 & 4521 & 0.20 & 8142 & 0.33 & 274.00 & 0.21 \\
\hline Alkylating agents & & 125811 & 6.64 & 132109 & 6.50 & 147076 & 6.39 & 148654 & 5.97 & 18.16 & -0.67 \\
\hline $\begin{array}{l}\text { Nitrogen mustard } \\
\text { analogues }\end{array}$ & $\begin{array}{l}\text { Cyclophosphamide, chlorambucil, melphalan, } \\
\text { ifosfamide, bendamustine }\end{array}$ & 112602 & 5.95 & 117101 & 5.76 & 125769 & 5.47 & 130042 & 5.22 & 15.49 & -0.72 \\
\hline Alkyl sulfonates & Busulfan & 301 & 0.02 & 279 & 0.01 & 318 & 0.01 & 255 & 0.01 & -15.28 & -0.01 \\
\hline Nitrosoureas & Carmustine & 250 & 0.01 & 218 & 0.01 & 263 & 0.01 & 321 & 0.01 & 28.40 & 0.00 \\
\hline Other alkylating agents & Temozolomide, dacarbazine & 12658 & 0.67 & 14511 & 0.71 & 20726 & 0.90 & 18036 & 0.72 & 42.49 & 0.06 \\
\hline Antimetabolites & & 911611 & 48.15 & 965096 & 47.47 & 1076871 & 46.81 & 1143596 & 45.93 & 25.45 & -2.22 \\
\hline Folic acid analogues & Methotrexate, pemetrexed & 316174 & 16.70 & 349463 & 17.19 & 386008 & 16.78 & 426480 & 17.13 & 34.89 & 0.43 \\
\hline Purine analogues & Mercaptopurine, cladribine, fludarabine & 12550 & 0.66 & 12094 & 0.59 & 12277 & 0.53 & 12891 & 0.52 & 2.72 & -0.15 \\
\hline Pyrimidine analogues & $\begin{array}{l}\text { Cytarabine, fluorouracil, tegafur, gemcitabine, } \\
\text { capecitabine, tegafur_combinations }\end{array}$ & 582887 & 30.78 & 603539 & 29.68 & 678586 & 29.50 & 704225 & 28.28 & 20.82 & -2.50 \\
\hline \multicolumn{2}{|c|}{ Plant alkaloids and other natural products } & 217347 & 11.48 & 222304 & 10.93 & 250312 & 10.88 & 250273 & 10.05 & 15.15 & -1.43 \\
\hline $\begin{array}{l}\text { Vinca alkaloids and } \\
\text { analogues }\end{array}$ & Vinblastine, vincristine, vinorelbine & 84009 & 4.44 & 85659 & 4.21 & 88135 & 3.83 & 88377 & 3.55 & 5.20 & -0.89 \\
\hline $\begin{array}{l}\text { Podophyllotoxin } \\
\text { derivatives }\end{array}$ & Etoposide & 28864 & 1.52 & 30188 & 1.48 & 32990 & 1.43 & 34587 & 1.39 & 19.83 & -0.14 \\
\hline Taxanes & Paclitaxel, docetaxel & 104474 & 5.52 & 106457 & 5.24 & 129187 & 5.62 & 127309 & 5.11 & 21.86 & -0.40 \\
\hline \multicolumn{2}{|c|}{ Cytotoxic antibiotics and related substances } & 140168 & 7.40 & 140697 & 6.92 & 145663 & 6.33 & 146796 & 5.90 & 4.73 & -1.51 \\
\hline Actinomycines & Dactinomycin & 616 & 0.03 & 698 & 0.03 & 667 & 0.03 & 761 & 0.03 & 23.54 & 0.00 \\
\hline $\begin{array}{l}\text { Anthracyclines and } \\
\text { related substances }\end{array}$ & $\begin{array}{l}\text { Doxorubicin, daunorubicin, epirubicin, } \\
\text { idarubicin, mitoxantrone }\end{array}$ & 99422 & 5.25 & 101826 & 5.01 & 107177 & 4.66 & 106499 & 4.28 & 7.12 & -0.97 \\
\hline $\begin{array}{l}\text { Other cytotoxic } \\
\text { antibiotics }\end{array}$ & Bleomycin, mitomycin & 40130 & 2.12 & 38173 & 1.88 & 37819 & 1.64 & 39536 & 1.59 & -1.48 & -0.53 \\
\hline $\begin{array}{l}\text { Other non-targeted } \\
\text { therapies }\end{array}$ & & 380316 & 20.09 & 422553 & 20.78 & 471677 & 20.50 & 494514 & 19.86 & 30.03 & -0.23 \\
\hline Platinum compounds & Cisplatin, carboplatin, oxaliplatin & 254636 & 13.45 & 286260 & 14.08 & 304437 & 13.23 & 306659 & 12.32 & 20.43 & -1.13 \\
\hline $\begin{array}{l}\text { Sensitisers used in } \\
\text { photodynamic/radiation } \\
\text { therapy }\end{array}$ & Verteporfin & 120 & 0.01 & 88 & 0.00 & 88 & 0.00 & 95 & 0.00 & -20.83 & 0.00 \\
\hline Others & $\begin{array}{l}\text { Asparaginase, hydroxycarbamide, estramustine, } \\
\text { tretinoin, topotecan, irinotecan, mitotane, } \\
\text { arsenic trioxide }\end{array}$ & 125560 & 6.63 & 136205 & 6.70 & 167152 & 7.27 & 187760 & 7.54 & 49.54 & 0.91 \\
\hline
\end{tabular}




\section{Drugs of interest}

We used the Anatomical Therapeutic Chemical (ATC) classification system developed by the WHO. We identified all antineoplastic agents using ATC codes 'L01'. Antineoplastic agents were grouped into six classes, based on the ATC system: (1) targeted therapies, including monoclonal antibodies (rituximab, trastuzumab, cetuximab), protein kinase inhibitors (imatinib, gefitinib, erlotinib, sunitinib, sorafenib, dasatinib, nilotinib, temsirolimus, everolimus, pazopanib) and bortezomib; these have all been used for the treatment of cancer in Taiwan; (2) alkylating agents (including nitrogen mustard analogues, alkyl sulfonates, nitrosoureas and other alkylating agents); (3) antimetabolites (including folic acid analogues, purine analogues and pyrimidine analogues); (4) plant alkaloids and other natural products (including Vinca alkaloids and analogues, podophyllotoxin derivatives and taxanes); (5) cytotoxic antibiotics and related substances (including actinomycines, anthracyclines and related substances and other cytotoxic antibiotics) and (6) other antineoplastic agents (including platinum compounds, sensitisers used in photodynamic/radiation therapy and other antineoplastic agents).

\section{Measurements}

To examine trends in use and costs of each class of antineoplastic agent (including targeted therapies), we calculated quarterly and yearly numbers of prescriptions and costs from 2009 to 2012. Then, for each class, we calculated the proportion of its use and costs among total use and total costs of all antineoplastic agents. For example, market share by prescription volume for targeted therapies was estimated by: number of prescriptions for targeted therapies divided by total number of prescriptions for all antineoplastic agents; and the market share by costs was estimated by: costs of targeted therapies divided by total costs of all antineoplastic agents. We also calculated cost per prescription for each class of antineoplastic agent.

To understand which cancers accounted for high use of targeted therapies, we first selected the 20 most common types of cancer in Taiwan, based on prevalence (see online supplementary appendix). We used the total prescription volume and total costs for targeted therapies in Taiwan as the denominator and conducted analyses using clinical indication of their use by type of cancer.

\section{Statistical analysis}

To assess the quarterly trends in market shares by prescription volume and costs of targeted therapies among all antineoplastic agents, we used a time series design with the Autoregressive Integrated Moving Average (ARIMA) model, which was developed by Box and Jenkins. ${ }^{44}$ The model is generally referred to as an
$\operatorname{ARIMA}(p, d, q)$ model where parameters $p, d$ and $q$ are non-negative integers that refer to the order of the autoregressive, integrated and moving average parts of the model, respectively. These models are fitted to time series data either to better understand the data or to determine points in the series. ${ }^{45}$ We used the estimated rates by ARIMA model for time series graphs. All analyses were carried out with SAS software, V.9.3 (SAS Institute, Cary, North Carolina, USA).

\section{RESULTS}

Between 2009 and 2012, prescriptions for antineoplastic agents grew $31.51 \%$ (an average rate of $10.5 \%$ increase per year) (table 1). By class, prescriptions for alkylating agents, antimetabolites, plant alkaloids and cytotoxic antibiotics increased in number during this period, but their market shares decreased: $-0.67 \%,-2.22 \%$, $-1.43 \%$ and $-1.51 \%$, respectively. In contrast, the market share of targeted therapies grew from $6.24 \%$ in 2009 to $12.29 \%$ in 2012. Specifically, market shares of monoclonal antibodies and protein kinase inhibitors doubled, from $2.75 \%$ to $5.79 \%$ and from $3.38 \%$ to
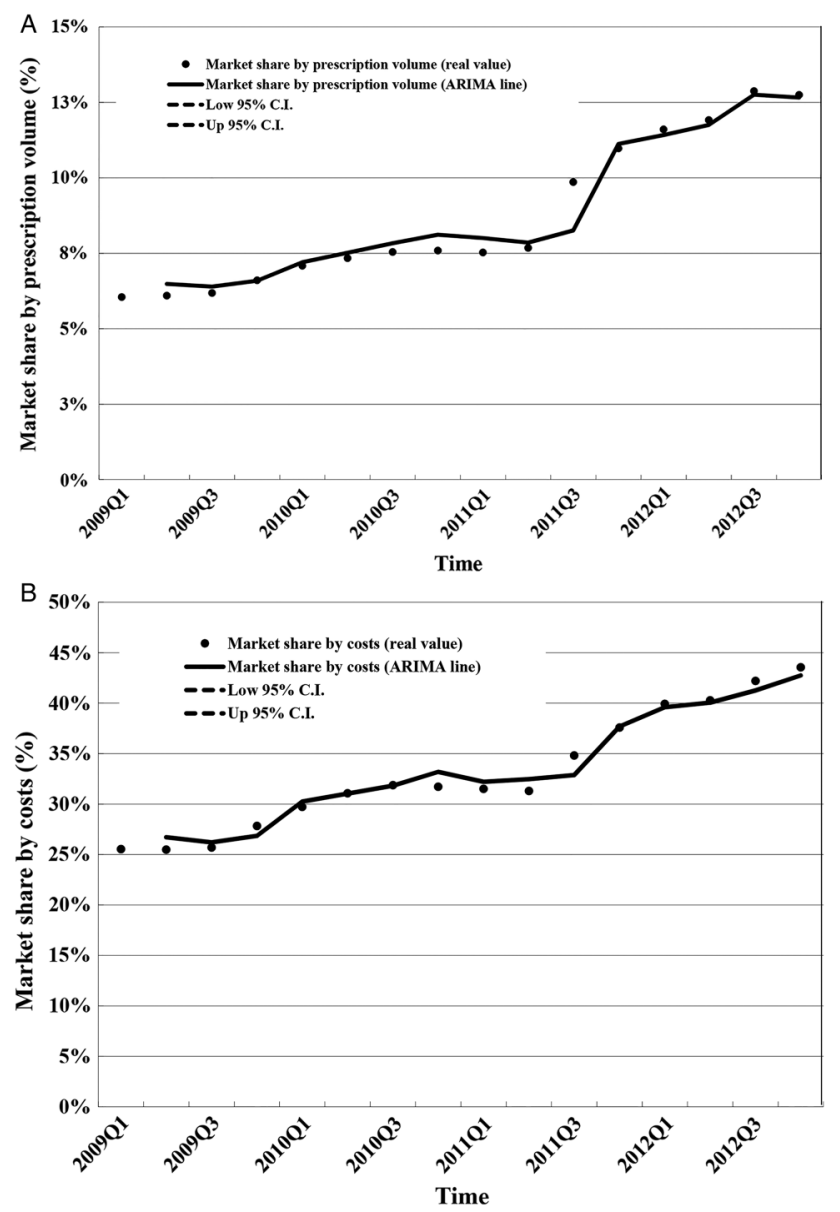

Figure 1 The 2009-2012 trends in market shares by prescription volume $(A)$ and costs $(B)$ for targeted therapies. (A) Market share by prescription volume. (B) Market share by costs. ARIMA, Autoregressive Integrated Moving Average. 
Table 2 Costs of antineoplastic agents in Taiwan (2009-2012)

Cost (market share by costs)

2009

2010

2011

2012

2009-2012

\begin{tabular}{|c|c|c|c|c|c|c|c|c|c|c|c|}
\hline \multirow[b]{2}{*}{ Drug class } & \multirow[b]{2}{*}{$\begin{array}{l}\text { Drug name for patients } \\
\text { with cancer }\end{array}$} & & & & & & \\
\hline & & Cost (US\$) & $\begin{array}{l}\text { Per } \\
\text { cent }\end{array}$ & Cost (US\$) & $\begin{array}{l}\text { Per } \\
\text { cent }\end{array}$ & Cost (US\$) & $\begin{array}{l}\text { Per } \\
\text { cent }\end{array}$ & Cost (US\$) & $\begin{array}{l}\text { Per } \\
\text { cent }\end{array}$ & $\begin{array}{l}\text { Growth rate } \\
\text { of } N(\%)\end{array}$ & $\begin{array}{l}\text { Growth rate } \\
\text { of market } \\
\text { share (\%) }\end{array}$ \\
\hline $\begin{array}{l}\text { All antineoplastic } \\
\text { agents }\end{array}$ & & 491387822 & 100 & 570369759 & 100 & 660138086 & 100 & 740386783 & 100 & 50.67 & \\
\hline Targeted & & 128541502 & 26.16 & 177668722 & 31.15 & 224327855 & 33.98 & 307754974 & 41.57 & 139.42 & 15.41 \\
\hline \multicolumn{12}{|c|}{ - le } \\
\hline $\begin{array}{l}\text { Monoclonal } \\
\text { antibodies }\end{array}$ & $\begin{array}{l}\text { Rituximab, trastuzumab, } \\
\text { cetuximab, bevacizumab }\end{array}$ & 71869602 & 14.63 & 104739673 & 18.36 & 137951386 & 20.90 & 176477405 & 23.84 & 145.55 & 9.21 \\
\hline $\begin{array}{l}\text { Protein kinase } \\
\text { inhibitors }\end{array}$ & $\begin{array}{l}\text { Imatinib, gefitinib, erlotinib, } \\
\text { sunitinib, sorafenib, dasatinib, } \\
\text { nilotinib, temsirolimus, } \\
\text { everolimus, pazopanib }\end{array}$ & 52651186 & 10.71 & 67484747 & 11.83 & 79001874 & 11.97 & 119383796 & 16.12 & 126.74 & 5.41 \\
\hline $\begin{array}{l}\text { Other targeted } \\
\text { therapy agents }\end{array}$ & Bortezomib & 4020714 & 0.82 & 5444303 & 0.95 & 7374595 & 1.12 & 11893774 & 1.61 & 195.81 & 0.79 \\
\hline Alkylating agents & & 15551932 & 3.16 & 17481103 & 3.06 & 18968906 & 2.87 & 18999092 & 2.57 & 22.17 & -0.60 \\
\hline $\begin{array}{l}\text { Nitrogen } \\
\text { mustard } \\
\text { analogues }\end{array}$ & $\begin{array}{l}\text { Cyclophosphamide, } \\
\text { chlorambucil, melphalan, } \\
\text { ifosfamide, bendamustine }\end{array}$ & 4495217 & 0.91 & 4897936 & 0.86 & 4878608 & 0.74 & 4261046 & 0.58 & -5.21 & -0.34 \\
\hline Alkyl sulfonates & Busulfan & 374465 & 0.08 & 454805 & 0.08 & 460163 & 0.07 & 488410 & 0.07 & 30.43 & -0.01 \\
\hline Nitrosoureas & Carmustine & 41620 & 0.01 & 43440 & 0.01 & 153101 & 0.02 & 392128 & 0.05 & 842.16 & 0.04 \\
\hline $\begin{array}{l}\text { Other alkylating } \\
\text { agents }\end{array}$ & Temozolomide, dacarbazine & 10640629 & 2.17 & 12084922 & 2.12 & 13477034 & 2.04 & 13857508 & 1.87 & 30.23 & -0.29 \\
\hline Antimetabolites & & 96951076 & 19.73 & 117122829 & 20.53 & 128199087 & 19.42 & 131805930 & 17.80 & 35.95 & -1.93 \\
\hline $\begin{array}{l}\text { Folic acid } \\
\text { analogues }\end{array}$ & Methotrexate, pemetrexed & 31305924 & 6.37 & 50705521 & 8.89 & 61101669 & 9.26 & 66069402 & 8.92 & 111.04 & 2.55 \\
\hline $\begin{array}{l}\text { Purine } \\
\text { analogues }\end{array}$ & $\begin{array}{l}\text { Mercaptopurine, cladribine, } \\
\text { fludarabine }\end{array}$ & 304010 & 0.06 & 265754 & 0.05 & 365404 & 0.06 & 311619 & 0.04 & 2.50 & -0.02 \\
\hline $\begin{array}{l}\text { Pyrimidine } \\
\text { analogues }\end{array}$ & $\begin{array}{l}\text { Cytarabine, fluorouracil, } \\
\text { tegafur, gemcitabine, } \\
\text { capecitabine, } \\
\text { tegafur_combinations }\end{array}$ & 65341142 & 13.30 & 66151554 & 11.60 & 66732014 & 10.11 & 65424909 & 8.84 & 0.13 & -4.46 \\
\hline $\begin{array}{l}\text { Plant alkaloids } \\
\text { and other natural } \\
\text { products }\end{array}$ & & 79509189 & 16.18 & 72920907 & 12.78 & 84694476 & 12.83 & 86583703 & 11.69 & 8.90 & -4.49 \\
\hline $\begin{array}{l}\text { Vinca alkaloids } \\
\text { and analogues }\end{array}$ & $\begin{array}{l}\text { Vinblastine, vincristine, } \\
\text { vinorelbine }\end{array}$ & 20326687 & 4.14 & 22006619 & 3.86 & 23924553 & 3.62 & 25170345 & 3.40 & 23.83 & -0.74 \\
\hline $\begin{array}{l}\text { Podophyllotoxin } \\
\text { derivatives }\end{array}$ & Etoposide & 2164352 & 0.44 & 1643415 & 0.29 & 1651811 & 0.25 & 1579203 & 0.21 & -27.04 & -0.23 \\
\hline Taxanes & Paclitaxel, docetaxel & 57018150 & 11.60 & 49270873 & 8.64 & 59118111 & 8.96 & 59834155 & 8.08 & 4.94 & -3.52 \\
\hline
\end{tabular}




\begin{tabular}{|c|c|c|c|c|c|c|c|c|c|c|c|}
\hline \multirow[b]{3}{*}{ Drug class } & \multirow[b]{3}{*}{$\begin{array}{l}\text { Drug name for patients } \\
\text { with cancer }\end{array}$} & \multicolumn{8}{|c|}{ Cost (market share by costs) } & \multirow{2}{*}{\multicolumn{2}{|c|}{ 2009-2012 }} \\
\hline & & 2009 & & 2010 & & 2011 & & 2012 & & & \\
\hline & & Cost (US\$) & $\begin{array}{l}\text { Per } \\
\text { cent }\end{array}$ & Cost (US\$) & $\begin{array}{l}\text { Per } \\
\text { cent }\end{array}$ & Cost (US\$) & $\begin{array}{l}\text { Per } \\
\text { cent }\end{array}$ & Cost (US\$) & $\begin{array}{l}\text { Per } \\
\text { cent }\end{array}$ & $\begin{array}{l}\text { Growth rate } \\
\text { of } N(\%)\end{array}$ & $\begin{array}{l}\text { Growth rate } \\
\text { of market } \\
\text { share (\%) }\end{array}$ \\
\hline $\begin{array}{l}\text { Cytotoxic } \\
\text { antibiotics and } \\
\text { related substances }\end{array}$ & & 26190529 & 5.33 & 26232768 & 4.60 & 27270661 & 4.13 & 26075058 & 3.52 & -0.44 & -1.81 \\
\hline Actinomycines & Dactinomycin & 16854 & 0.00 & 18603 & 0.00 & 18303 & 0.00 & 19062 & 0.00 & 13.10 & 0.00 \\
\hline $\begin{array}{l}\text { Anthracyclines } \\
\text { and related } \\
\text { substances }\end{array}$ & $\begin{array}{l}\text { Doxorubicin, daunorubicin, } \\
\text { epirubicin, idarubicin, } \\
\text { mitoxantrone }\end{array}$ & 24489365 & 4.98 & 24531634 & 4.30 & 25576627 & 3.87 & 24215313 & 3.27 & -1.12 & -1.71 \\
\hline $\begin{array}{l}\text { Other cytotoxic } \\
\text { antibiotics }\end{array}$ & Bleomycin, mitomycin & 1684311 & 0.34 & 1682531 & 0.29 & 1675731 & 0.25 & 1840682 & 0.25 & 9.28 & -0.09 \\
\hline $\begin{array}{l}\text { Other } \\
\text { non-targeted } \\
\text { therapies }\end{array}$ & & 144643593 & 29.44 & 158943430 & 27.87 & 176677101 & 26.76 & 169168026 & 22.85 & 16.96 & -6.59 \\
\hline $\begin{array}{l}\text { Platinum } \\
\text { compounds }\end{array}$ & $\begin{array}{l}\text { Cisplatin, carboplatin, } \\
\text { oxaliplatin }\end{array}$ & 50363294 & 10.25 & 53988423 & 9.47 & 52077277 & 7.89 & 35697261 & 4.82 & -29.12 & -5.43 \\
\hline $\begin{array}{l}\text { Sensitisers used } \\
\text { in } \\
\text { photodynamic/ } \\
\text { radiation therapy }\end{array}$ & Verteporfin & 169600 & 0.03 & 124373 & 0.02 & 124373 & 0.02 & 134267 & 0.02 & -20.83 & -0.02 \\
\hline Others & $\begin{array}{l}\text { Asparaginase, } \\
\text { hydroxycarbamide, } \\
\text { estramustine, tretinoin, } \\
\text { topotecan, irinotecan, } \\
\text { mitotane, arsenic trioxide }\end{array}$ & 94110699 & 19.15 & 104830634 & 18.38 & 124475451 & 18.86 & 133336498 & 18.01 & 41.68 & -1.14 \\
\hline
\end{tabular}


$6.18 \%$, respectively. Figure 1A shows ARIMA regression estimated quarterly trends in market share by prescription volume for targeted therapies during the study period.

Table 2 presents the costs for all and each type of antineoplastic drug between 2009 and 2012. There was a large growth in total costs of antineoplastic agents from 2009 to 2012 (an overall increase of 50.67\%, an average rate of $16.89 \%$ increase per year). By class, the yearly market share by costs for alkylating agents, antimetabolites, plant alkaloids and cytotoxic antibiotics, reduced by $0.60 \%, 1.93 \%, 4.49 \%$ and $1.81 \%$, from 2009 to 2012 . In contrast, annual costs of targeted therapies grew from US $\$ 129$ million (26.16\% of all costs for antineoplastic agents) in 2009 to US\$308 million (41.57\%) in 2012. Specifically, the market share by costs for monoclonal antibodies and protein kinase inhibitors increased from $14.63 \%$ to $23.84 \%$ and from $10.71 \%$ to $16.12 \%$, respectively. Figure 1B shows the ARIMA regression estimated quarterly trend in market share by costs for targeted therapies during the study period.

Table 3 shows the cost per prescription for each class of antineoplastic agents between 2009 and 2012. We found that, in 2012, targeted therapies had the highest cost per prescription (US\$1005), other antineoplastic agents in descending order by cost per prescription were plant alkaloids and other natural products (US\$346), other non-targeted therapies (US\$342), cytotoxic antibiotics and related substances (US\$178), alkylating agents (US\$128) and antimetabolites (US \$115). There was about a 3-fold difference in cost per prescription between targeted therapies, and plant alkaloids and other natural products, and about a 10-fold difference between targeted therapies and antimetabolites.

Figure 2A, B presents the distribution ratios of targeted therapy use for 20 cancers during 2009-2012. Table 4 shows the yearly distribution ratios of targeted

Table 3 Cost per prescription of antineoplastic agents in Taiwan (2009-2012)

\begin{tabular}{|c|c|c|c|c|c|}
\hline \multirow[b]{2}{*}{ Drug class } & \multirow[b]{2}{*}{ Drug name for patients with cancer } & \multicolumn{4}{|c|}{ Cost per prescription (US\$) } \\
\hline & & 2009 & 2010 & 2011 & 2012 \\
\hline \multicolumn{2}{|l|}{ All antineoplastic agents } & 260 & 281 & 287 & 297 \\
\hline \multicolumn{2}{|l|}{ Targeted therapies } & 1088 & 1181 & 1073 & 1005 \\
\hline Monoclonal antibodies & Rituximab, trastuzumab, cetuximab, bevacizumab & 1380 & 1527 & 1351 & 1224 \\
\hline Protein kinase inhibitors & $\begin{array}{l}\text { Imatinib, gefitinib, erlotinib, sunitinib, sorafenib, dasatinib, } \\
\text { nilotinib, temsirolimus, everolimus, pazopanib }\end{array}$ & 823 & 858 & 771 & 776 \\
\hline $\begin{array}{l}\text { Other targeted therapy } \\
\text { agents }\end{array}$ & bortezomib & 1847 & 1739 & 1631 & 1461 \\
\hline \multicolumn{2}{|l|}{ Alkylating agents } & 124 & 132 & 129 & 128 \\
\hline Nitrogen mustard analogues & $\begin{array}{l}\text { Cyclophosphamide, chlorambucil, melphalan, ifosfamide, } \\
\text { bendamustine }\end{array}$ & 40 & 42 & 39 & 33 \\
\hline Alkyl sulfonates & Busulfan & 1244 & 1630 & 1447 & 1915 \\
\hline Nitrosoureas & Carmustine & 166 & 199 & 582 & 1222 \\
\hline Other alkylating agents & Temozolomide, dacarbazine & 841 & 833 & 650 & 768 \\
\hline \multicolumn{2}{|l|}{ Antimetabolites } & 106 & 121 & 119 & 115 \\
\hline Folic acid analogues & Methotrexate, pemetrexed & 99 & 145 & 158 & 155 \\
\hline Purine analogues & Mercaptopurine, cladribine, fludarabine & 24 & 22 & 30 & 24 \\
\hline Pyrimidine analogues & $\begin{array}{l}\text { Cytarabine, fluorouracil, tegafur, gemcitabine, } \\
\text { capecitabine, tegafur_combinations }\end{array}$ & 112 & 110 & 98 & 93 \\
\hline \multicolumn{2}{|c|}{ Plant Alkaloids and other Natural Products } & 366 & 328 & 338 & 346 \\
\hline $\begin{array}{l}\text { Vinca alkaloids and } \\
\text { analogues }\end{array}$ & Vinblastine, vincristine, vinorelbine & 242 & 257 & 271 & 285 \\
\hline Podophyllotoxin derivatives & Etoposide & 75 & 54 & 50 & 46 \\
\hline Taxanes & Paclitaxel, docetaxel & 546 & 463 & 458 & 470 \\
\hline \multicolumn{2}{|c|}{ Cytotoxic antibiotics and related substances } & 187 & 186 & 187 & 178 \\
\hline Actinomycines & Dactinomycin & 27 & 27 & 27 & 25 \\
\hline $\begin{array}{l}\text { Anthracyclines and related } \\
\text { substances }\end{array}$ & $\begin{array}{l}\text { Doxorubicin, daunorubicin, epirubicin, idarubicin, } \\
\text { mitoxantrone }\end{array}$ & 246 & 241 & 239 & 227 \\
\hline Other cytotoxic antibiotics & Bleomycin, mitomycin & 42 & 44 & 44 & 47 \\
\hline \multicolumn{2}{|l|}{ Other non-targeted therapies } & 380 & 376 & 375 & 342 \\
\hline Platinum compounds & Cisplatin, carboplatin, oxaliplatin & 198 & 189 & 171 & 116 \\
\hline $\begin{array}{l}\text { Sensitisers used in } \\
\text { photodynamic/radiation } \\
\text { therapy }\end{array}$ & Verteporfin & 1413 & 1413 & 1413 & 1413 \\
\hline Others & $\begin{array}{l}\text { Asparaginase, hydroxycarbamide, estramustine, tretinoin, } \\
\text { topotecan, irinotecan, mitotane, arsenic trioxide }\end{array}$ & 750 & 770 & 745 & 710 \\
\hline
\end{tabular}



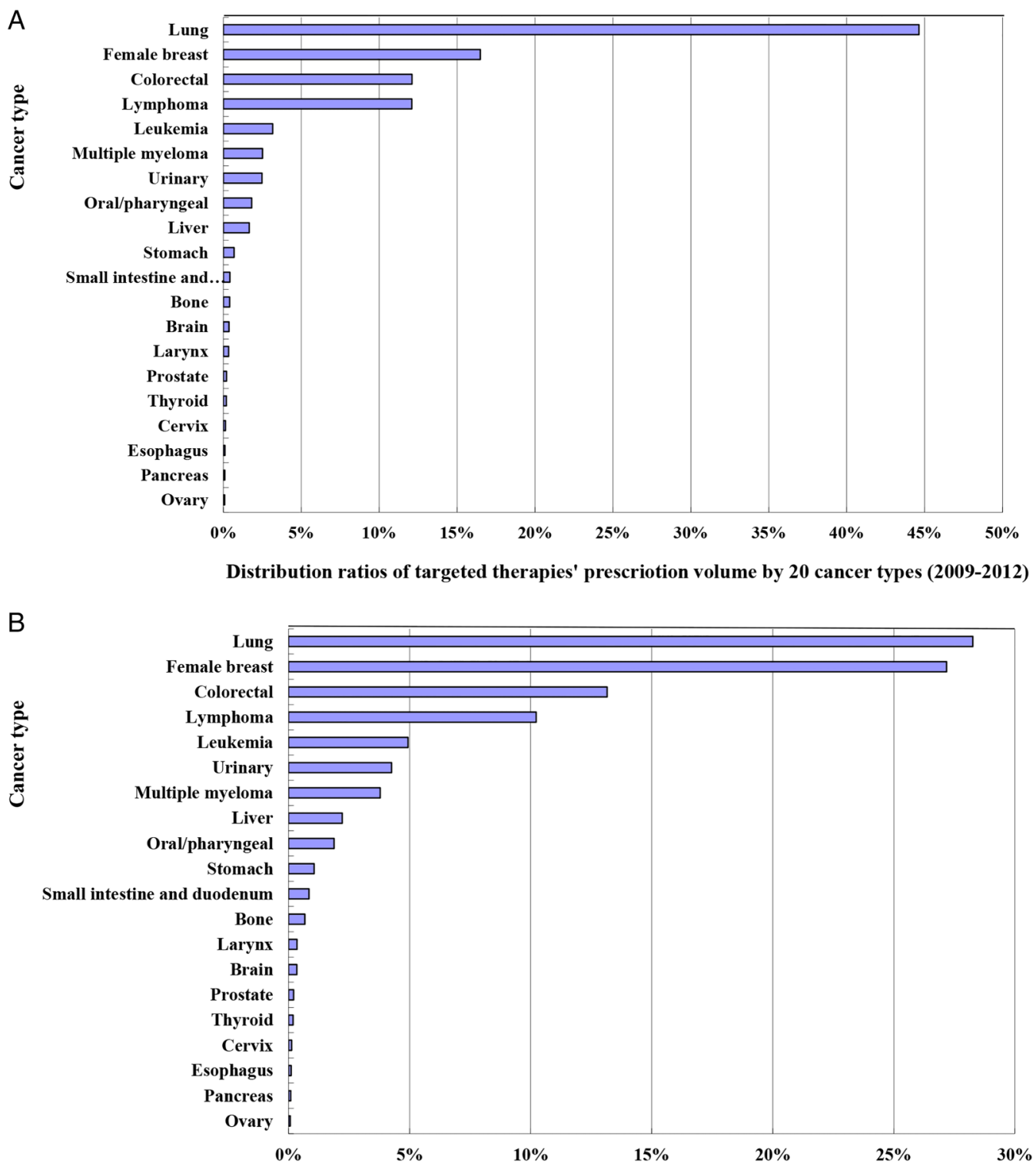

Distribution ratios of targeted therapies' costs by 20 cancer types (2009-2012)

Figure 2 Use (A) and costs (B) of targeted therapies by 20 cancer types (2009-2012). (A) Distribution ratios of prescription volume for targeted therapies by cancer type. (B) Distribution ratios of costs for targeted therapies by cancer type.

therapy use by cancer type over time. Our results showed that use and costs for targeted therapies differed substantially between different types of cancer. During 2009-2012, targeted therapies were mostly used for cancers of the lung and female breast, colorectal cancer, lymphoma and leukaemia, in order of volume. These five cancer types accounted for, respectively, $44.64 \%$, $16.49 \%, 12.11 \%, 12.09 \%$ and $3.17 \%$ of prescriptions for targeted therapies (together 88.5\%); and $28.26 \%$, $27.18 \%, 13.16 \%, 10.23 \%$ and $4.94 \%$ of costs for targeted therapies (together $83.77 \%$ ) among these 20 common cancer types.

\section{DISCUSSION}

To the best of our knowledge, this is the first study to examine the national trend in use and costs of targeted therapies for treatment of cancer in Taiwan. Our findings indicated that, compared with other classes of antineoplastic drugs, use of targeted therapies, novel agents for cancer treatment, increased substantially and is causing great economic burden in Taiwan. Cancers of the lung and female breast, and colorectal cancer, accounted for the most used targeted therapies.

Between 2009 and 2012, use and costs of targeted therapies increased almost threefold (tables 1 and 2), with steep growth since the third quarter of 2011 (figure 1). This trend is likely to continue in the future. We found that the average cost per prescription of targeted therapies was much higher than that of other classes of antineoplastic agents, with a 3-10-fold difference in 2012. It is important that policymakers revisit the pricing and reimbursement structures for these 


\begin{tabular}{|c|c|c|c|c|c|c|c|c|c|c|c|c|c|}
\hline & & \multicolumn{6}{|c|}{ Distribution ratio based on prescription volume (\%) } & \multicolumn{6}{|c|}{ Distribution ratio of costs (\%) } \\
\hline \multicolumn{2}{|c|}{ Cancer type } & 2009 & 2010 & 2011 & 2012 & $\begin{array}{l}\text { 2009-2012 } \\
\text { overall }\end{array}$ & $\begin{array}{l}2009-2012 \\
\text { growth rate }\end{array}$ & 2009 & 2010 & 2011 & 2012 & $\begin{array}{l}\text { 2009-2012 } \\
\text { overall }\end{array}$ & $\begin{array}{l}2009-2012 \\
\text { growth rate }\end{array}$ \\
\hline 01 & Lung & 51.87 & 45.94 & 43.03 & 42.18 & 44.64 & -9.68 & 39.55 & 28.79 & 25.48 & 25.24 & 28.26 & -14.32 \\
\hline 02 & Female breast & 11.71 & 18.62 & 18.47 & 15.95 & 16.49 & 4.24 & 20.91 & 31.45 & 30.60 & 24.78 & 27.18 & 3.87 \\
\hline 03 & Colorectal & 9.52 & 7.01 & 12.32 & 15.62 & 12.11 & 6.10 & 12.12 & 8.65 & 13.13 & 16.29 & 13.16 & 4.16 \\
\hline 04 & Lymphoma & 17.87 & 14.92 & 11.84 & 8.50 & 12.09 & -9.37 & 15.24 & 11.63 & 9.96 & 7.49 & 10.23 & -7.74 \\
\hline 05 & Liver & 0.24 & 0.23 & 0.28 & 3.93 & 1.66 & 3.69 & 0.25 & 0.22 & 0.28 & 5.65 & 2.22 & 5.40 \\
\hline 06 & Leukaemia & 1.75 & 2.81 & 3.66 & 3.57 & 3.17 & 1.83 & 2.50 & 4.80 & 5.60 & 5.56 & 4.94 & 3.06 \\
\hline 07 & Urinary & 0.43 & 2.70 & 3.01 & 2.82 & 2.48 & 2.39 & 0.52 & 4.53 & 5.25 & 4.95 & 4.26 & 4.43 \\
\hline 08 & Multiple myeloma & 1.92 & 2.11 & 2.39 & 3.07 & 2.52 & 1.15 & 3.37 & 3.19 & 3.60 & 4.45 & 3.79 & 1.08 \\
\hline 09 & Oral/pharyngeal & 1.58 & 2.40 & 2.01 & 1.48 & 1.82 & -0.10 & 1.59 & 2.43 & 2.03 & 1.57 & 1.88 & -0.03 \\
\hline 10 & Stomach & 0.97 & 0.77 & 0.62 & 0.58 & 0.69 & -0.39 & 1.28 & 1.14 & 1.04 & 0.93 & 1.06 & -0.35 \\
\hline 11 & Brain & 0.34 & 0.24 & 0.27 & 0.48 & 0.36 & 0.14 & 0.27 & 0.18 & 0.17 & 0.60 & 0.34 & 0.33 \\
\hline 12 & $\begin{array}{l}\text { Small intestine and } \\
\text { duodenum }\end{array}$ & 0.43 & 0.51 & 0.43 & 0.34 & 0.41 & -0.09 & 0.80 & 0.99 & 0.90 & 0.74 & 0.85 & -0.06 \\
\hline 13 & Bone & 0.45 & 0.48 & 0.44 & 0.33 & 0.41 & -0.12 & 0.65 & 0.74 & 0.80 & 0.57 & 0.68 & -0.08 \\
\hline 14 & Thyroid & 0.15 & 0.13 & 0.22 & 0.22 & 0.19 & 0.07 & 0.20 & 0.14 & 0.20 & 0.20 & 0.19 & 0.00 \\
\hline 15 & Prostate & 0.18 & 0.23 & 0.19 & 0.22 & 0.20 & 0.04 & 0.16 & 0.27 & 0.18 & 0.22 & 0.21 & 0.06 \\
\hline 16 & Larynx & 0.24 & 0.48 & 0.41 & 0.26 & 0.34 & 0.02 & 0.26 & 0.47 & 0.41 & 0.27 & 0.35 & 0.01 \\
\hline 17 & Pancreas & 0.01 & 0.02 & 0.10 & 0.14 & 0.09 & 0.13 & 0.01 & 0.02 & 0.06 & 0.20 & 0.10 & 0.19 \\
\hline 18 & Cervix & 0.16 & 0.20 & 0.14 & 0.11 & 0.14 & -0.05 & 0.16 & 0.17 & 0.13 & 0.10 & 0.13 & -0.05 \\
\hline 19 & Oesophagus & 0.09 & 0.12 & 0.13 & 0.09 & 0.11 & 0.01 & 0.07 & 0.12 & 0.13 & 0.10 & 0.11 & 0.03 \\
\hline \multirow[t]{2}{*}{20} & Ovary & 0.10 & 0.08 & 0.05 & 0.10 & 0.08 & 0.00 & 0.09 & 0.08 & 0.05 & 0.08 & 0.07 & -0.01 \\
\hline & Total & 100.00 & 100.00 & 100.00 & 100.00 & 100.00 & & 100.00 & 100.00 & 100.00 & 100.00 & 100.00 & \\
\hline
\end{tabular}


medicines because prices for all targeted therapies are high even for those that offer limited clinical benefits.

Our study adds to the literature stating that the availability and increasing use of innovative but expensive targeted therapies are major drivers of increases in pharmaceutical expenditures. ${ }^{25}{ }^{26}$ We showed that the costs of targeted therapies accounted for almost $42 \%$ of expenditures for all antineoplastic agents in Taiwan in 2012. Monoclonal antibodies and protein kinase inhibitors contributed the most $(23.84 \%$ and $16.12 \%$ of costs for antineoplastic agents). Targeted therapies also dominate cancer drug expenditures in other countries, for example, they accounted for $63 \%$ of all cancer drug expenditures in 2011 in the commercially insured US population. ${ }^{46}$

The high cost of targeted therapies is a barrier to access targeted therapies for treatment of cancer. ${ }^{28}$ It is important to ensure patient access to effective targeted therapies without overspending the healthcare budget, given their clinical benefits. Many experts propose that dialogue involving all parties concerned (eg, policymakers, industry, clinicians, patients and the general public) is needed to address the reasons behind high prices of cancer drugs and to provide solutions to reduce prices. Experts also propose that drug prices should reflect objective measures of benefit, but should not exceed values that could harm patients and societies. ${ }^{27} 47$ Overall, strategies for future management of new cancer medicines might include raising the bar for clinical trials by defining clinically meaningful outcomes ${ }^{48}$ establishing minimum effectiveness levels for new cancer medicines, ${ }^{15} 24$ generating a list of essential medicines for patients with cancer, discussing potential future measures to fund new innovative cancer medicines without potentially compromising patients/healthcare systems, ${ }^{23}$ and determining the proportion of healthcare resources spent on cancer medicines based on the consideration of their balance of costs and outcomes. ${ }^{47}$

There are some limitations to this study. First, this study aimed to examine recent trends in drug utilisation and expenditures for cancer treatment and to estimate the market shares by prescription volume and costs for targeted therapies in Taiwan; our analysis only examined data up to 2012, as these were the more recent data available at the time of the analysis. We used aggregate data and did not analyse patient-level data, to understand the influence of patient characteristics on treatment selection and clinical outcomes of treatments. Additionally, this study did not examine the complex patterns of drug use, such as use of combination treatments, and targeted therapy adherence and persistence, again because of the lack of patient-level data. This study examined economic burden of targeted therapies from the perspective of the healthcare system; we did not examine patient contribution to drug costs in Taiwan; this warrants a separate study. Further, we did not characterise changes in the policy environment in Taiwan during the study period. Examples include the launch of new, competing targeted therapies, publication of large randomised clinical trial results, changes in clinical guidelines or reimbursement policies, and patient and provider factors (eg, patient clinical history, physician's knowledge and preference). Future studies are needed to examine the impact of changes in policy and the clinical environment, on use of targeted therapies. Finally, various types of restrictions (eg, prior authorisations) have been applied for many high-cost targeted therapies for cancer in Taiwan. ${ }^{49}$ How these restrictions impact cancer care and outcomes should be studied.

\section{CONCLUSION}

Targeted therapies have played an increasing and more important role in treatment of all malignancies in Taiwan, and are likely to pose substantial economic burden in the future. Cancers of the lung and female breast, and colorectal cancer, were identified as the main drivers of use and costs of targeted therapies in recent years. Policymakers, industry, clinicians and patients need to communicate and develop strategies to enable access to effective (and cost-effective) targeted therapies without overspending the healthcare budget.

Contributors $\mathrm{JCH}$ and $\mathrm{CYL}$ conceptualised and designed the study. JCH collected data, performed analysis, and drafted the manuscript. CYL reviewed all data and revised the manuscript critically for intellectual content. All the authors approved the final version for submission.

Funding JCH was supported by grants from Taiwan Food and Drug Administration (grant ID 104TFDA-JFDA-306) and Taiwan's Ministry of Science and Technology (grant ID MOST 104-2320-B-006-005).

Disclaimer The funders had no role in study design, data collection and analysis, decision to publish, or preparation of the manuscript.

Competing interests None declared.

Ethics approval National Cheng Kung University Hospital.

Provenance and peer review Not commissioned; externally peer reviewed.

Data sharing statement The authors have obtained nationwide, monthly claims data for cancer-related antineoplastic agents, from 2009 to 2012, from the Taiwan National Health Insurance Research Database (NHIRD). NHIRD does not permit external sharing of any of the data elements.

Open Access This is an Open Access article distributed in accordance with the Creative Commons Attribution Non Commercial (CC BY-NC 4.0) license, which permits others to distribute, remix, adapt, build upon this work noncommercially, and license their derivative works on different terms, provided the original work is properly cited and the use is non-commercial. See: http:// creativecommons.org/licenses/by-nc/4.0/

\section{REFERENCES}

1. Dranitsaris G, Truter I, Lubbe MS, et al. Advances in cancer therapeutics and patient access to new drugs. Pharmacoeconomics 2011;29:213-24.

2. Schoenlein PV, Hou M, Samaddar JS, et al. Downregulation of retinoblastoma protein is involved in the enhanced cytotoxicity of 4-hydroxytamoxifen plus mifepristone combination therapy versus antiestrogen monotherapy of human breast cancer. Int $J$ Oncol 2007;31:643-55.

3. Taiwan Health Promotion Administration, Ministry of Health and Welfare, 2011 Cancer Registry Annual Report, 2014. 
4. Wu CY, Lin JT. The changing epidemiology of Asian digestive cancers: from etiologies and incidences to preventive strategies. Best Pract Res Clin Gastroenterol 2015;29:843-53.

5. Biswas M, Ades AE, Hamilton W. Symptom lead times in lung and colorectal cancers: what are the benefits of symptom-based approaches to early diagnosis? Br J Cancer 2015;112:271-7.

6. Eeles RA, Morden JP, Gore M, et al. Adjuvant hormone therapy may improve survival in epithelial ovarian cancer: results of the AHT randomized trial. J Clin Oncol 2015;33:4138-44.

7. Schiller $\mathrm{JH}$, Harrington $\mathrm{D}$, Belani $\mathrm{CP}$, et al. Comparison of four chemotherapy regimens for advanced non-small-cell lung cancer. N Engl J Med 2002;346:92-8.

8. National Cancer Institute, Targeted Cancer Therapies. http://www. cancer.gov/about-cancer/treatment/types/targeted-therapies/ targeted-therapies-fact-sheet (accessed 13 Apr 2016).

9. Kohne $\mathrm{CH}$, Lenz HJ. Chemotherapy with targeted agents for the treatment of metastatic colorectal cancer. Oncologist 2009;14:478-88.

10. Mahalingam D, Mita A, Mita MM, et al. Targeted therapy for advanced non-small cell lung cancers: historical perspective, current practices, and future development. Curr Probl Cancer 2009;33:73-111.

11. Mok TS, Wu YL, Thongprasert S, et al. Gefitinib or carboplatin-paclitaxel in pulmonary adenocarcinoma. $N$ Engl J Med 2009;361:947-57.

12. Lu CY, Srasuebkul P, Drew AK, et al. Trastuzumab therapy in Australia: which patients with HER2+ metastatic breast cancer are assessed for cardiac function? Breast 2013:22:482-7.

13. Lu CY, Srasuebkul P, Drew AK, et al. Positive spillover effects of prescribing requirements: increased cardiac testing in patients treated with trastuzumab for HER2+ metastatic breast cancer. Intern Med J 2012;42:1229-35.

14. Weingart SN, Brown E, Bach PB, et al. NCCN task force report: oral chemotherapy. J Natl Compr Canc Netw 2008;6(Suppl 3): S1-14.

15. Kantarjian HM, Fojo T, Mathisen M, et al. Cancer drugs in the United States: Justum Pretium-the just price. J Clin Oncol 2013;31:3600-4.

16. Lu CY, Cohen JP. Can genomic medicine improve financial sustainability of health systems? Mol Diagn Ther 2015;19:71-7.

17. Lu CY, Williams K, Day R, et al. Access to high cost drugs in Australia. BMJ 2004;329:415-16.

18. Hall WD, Ward R, Liauw WS, et al. Tailoring access to high cost, genetically targeted drugs. Med J Aust 2005;182:607-8.

19. Mullard A. 2011 FDA drug approvals. Nat Rev Drug Discov 2012;11:91-4.

20. Godman B, Malmstrom RE, Diogene E, et al. Are new models needed to optimize the utilization of new medicines to sustain healthcare systems? Expert Rev Clin Pharmacol 2015;8:77-94.

21. Kelly RJ, Smith TJ. Delivering maximum clinical benefit at an affordable price: engaging stakeholders in cancer care. Lancet Oncol 2014;15:e112-18.

22. Howard DH, Bach PB, Berndt ER, et al. Pricing in the market for anticancer drugs. J Econ Perspect 2015;29:139-62.

23. Ghinea N, Kerridge I, Lipworth W. If we don't talk about value, cancer drugs will become terminal for health systems. The conversation, 2015. https://theconversation.com/if-we-dont-talkabout-value-cancer-drugs-will-become-terminal-for-health-systems44072

24. Ferguson JS, Summerhayes M, Masters S, et al. New treatments for advanced cancer: an approach to prioritization. $\mathrm{Br} J$ Cancer 2000;83:1268-73.

25. Karaca-Mandic P, McCullough JS, Siddiqui MA, et al. Impact of new drugs and biologics on colorectal cancer treatment and costs. $J$ Oncol Pract 2011;7(3 Suppl):e30s-7s.

26. Warren JL, Yabroff KR, Meekins A, et al. Evaluation of trends in the cost of initial cancer treatment. J Natl Cancer Inst 2008;100:888-97.
27. Experts in Chronic Myeloid Leukemia. The price of drugs for chronic myeloid leukemia $(C M L)$ is a reflection of the unsustainable prices of cancer drugs: from the perspective of a large group of CML experts. Blood 2013:121:4439-42.

28. O'Dowd A. Watchdog set to reject four drugs for kidney cancer on the NHS. BMJ 2008;337:a1262.

29. Campbell B, Morris R, Mandava L, et al. Identifying and selecting new procedures for health technology assessment: a decade of nice experience in the United Kingdom. Int J Technol Assess Health Care 2014;30:454-60.

30. Yue J, Tabloski P, Dowal SL, et al. NICE to HELP: operationalizing National Institute for Health and Clinical Excellence guidelines to improve clinical practice. J Am Geriatr Soc 2014;62:754-61.

31. Streat S, Munn S. Health economics and health technology assessment: perspectives from Australia and New Zealand. Crit Care Clin 2012;28:125-33, vii.

32. Chim L, Kelly PJ, Salkeld G, et al. Are cancer drugs less likely to be recommended for listing by the Pharmaceutical Benefits Advisory Committee in Australia? Pharmacoeconomics 2010;28:463-75.

33. Lu CY, Lupton C, Rakowsky S, et al. Patient access schemes in Asia-pacific markets: current experience and future potential. J Pharm Policy Pract 2015;8:6.

34. Faden RR, Chalkidou K, Appleby J, et al. Expensive cancer drugs: a comparison between the United States and the United Kingdom. Milbank Q 2009;87:789-819.

35. Vitry A, Roughead E. Managed entry agreements for pharmaceuticals in Australia. Health Policy 2014;117:345-52.

36. Ferrario A, Kanavos P. Dealing with uncertainty and high prices of new medicines: a comparative analysis of the use of managed entry agreements in Belgium, England, the Netherlands and Sweden. Soc Sci Med 2015;124:39-47.

37. Paris V, Belloni A. Value in Pharmaceutical Pricing. OECD Health Working Papers, No 63: OECD Publishing, 2013.

38. Yang BM. The future of health technology assessment in healthcare decision making in Asia. Pharmacoeconomics 2009;27: 891-901.

39. Oortwijn W, Mathijssen J, Banta D. The role of health technology assessment on pharmaceutical reimbursement in selected middleincome countries. Health Policy 2010;95:174-84.

40. Hsu JC, Lu CY. The evolution of Taiwan's National Health Insurance drug reimbursement scheme. Daru 2015;23:15.

41. National Health Insurance Administration, Directions of Drug Restricted Benefit for National Health Insurance. 2013. http://www. nhi.gov.tw/webdata/webdata.aspx?menu=21\&menu id=713\&webdata id=2919

42. Insurance BoNH. National Health Insurance Annual Statistical Report. October 2004. http://www.nhi.gov.tw/Resource/ webdata/ Attach_8661_1_s92.pdf (accessed 8 Jun 2011).

43. Liu SZ, Romeis JC. Assessing the effect of Taiwan's outpatient prescription drug copayment policy in the elderly. Med Care 2003; $41: 1331-42$

44. Mills TC. Time series techniques for economists. Cambridge University Press, 1990.

45. Asteriou DH, Stephen G. ARIMA Models and the Box-Jenkins Methodology, Applied Econometrics. 2nd edn. Palgrave MacMillan, 2011:265-86.

46. Shih YCT, Smieliauskas F, Geynisman DM, et al. Trends in the cost and use of targeted cancer therapies for the privately insured nonelderly: 2001 to 2011. J Clin Oncol 2015;33:2190-U232.

47. Jönsson B, Ramsey S, Wilking N. Cost effectiveness in practice and its effect on clinical outcomes. J Cancer Policy 2014;2:12-21.

48. Ellis LM, Bernstein DS, Voest EE, et al. American Society of Clinical Oncology perspective: raising the bar for clinical trials by defining clinically meaningful outcomes. J Clin Oncol 2014;32: 1277-80.

49. Taiwan National Health Insurance Administration, Schemes for National Health Insurance Drug Reimbursement System, 2014 